\title{
Remote Preparation of Mixed States via Noisy Entanglement
}

\author{
Guo-Yong Xiang, Jian Li, Bo Yu, and Guang-Can Guo \\ Key Laboratory of Quantum Information, University of Science and Technology \\ of China, CAS, Hefei 230026, People's Republic of China
}

\begin{abstract}
We present a practical and general scheme of remote preparation for pure and mixed state, which is proposed with an auxiliary qubit and controlled-NOT gate. We discuss the remote state preparation (RSP) in two important types of decoherent channel (depolarizing and dephasing). We realize RSP in the dephasing channel in our experiment by using spontaneous parametric down conversion (SPDC), linear optical elements and single photon detector. Our experiment results match the theoretical prediction well.
\end{abstract}

PACS numbers: 03.67.Hk; 03.65.Ud

\section{INTRODUCTION}

A principal goal of quantum information theory is to understand the resources necessary and sufficient for intact transmission of quantum states [1]. Obviously, Alice (the sender) can either physically send the particle (which is not interesting) or send a double infinity of bits of information across a classical channel to Bob. However, quantum teleportation 2] has provided us an interesting way to transmitting an arbitary quantum state using one maximal-entangled state (1 ebit) and two classical bits information $(2 \mathrm{cbits})$. Recently, remote state preparation (RSP) 3, 4, 5, 6, 1, 8, 9, 10, 11, 12] attracts attention of many scientists. RSP provides us a simple method to transmit pure quantum states using entanglement and classical communication when the sender knows the transmitted state and the receiver has partial knowledge of it. RSP protocols more economical than teleportation were found for certain ensembles [3, 4, 5, 6, 7, 8, 10, 12. The resources of 1 ebit and 2 cbits are sufficient and necessary for teleportation, while it can be reduced to 1 ebit and 1 cbit in asymptotics for remote state preparation. Such trade-off has been extent among resources of noiseless classical channels, noiseless quantum channels and maximally-entangled states in the generalized remote state preparation [13]. An experiment of RSP has been demonstrated in nuclear magnetic resonance (NMR) systems $[\underline{9}]$.

Recently, the RSP protocol is generalized from pure states to mixed ones. The essence is to replace von Neumann measurement by positive operator-valued measure (POVM) 7]. Here we present a scheme for remote preparation of mixed states (including pure ones) and realize it for the polarization states of single photons.

All protocols about RSP above is (deterministic or probabilistic) exact, in which state that Bob gets is as same as the one Alice wants to prepare. It is based on the maximally-entangled state shared between the two sides. However, entanglement is fragile under the interaction with environment. In this paper, two types of decoherence, depolarizing and dephasing, are discussed by using the state fidelity in theory as well as in our experiment.

This paper is organized as following. A RSP scheme of mixed states is presented in section II. The effect of decoherence is discussed with state fidelity in section III. In section IV, we carry out an experiment to remotely prepare pure and mixed states with dephaseing noisy entanglement. And a conclusion will be given in section V.

\section{REMOTE PREPARATION OF MIXED STATES}

An arbitrary state of single qubit can be represented as a vector $\vec{r}$ on or inside the Bloch sphere (see Fig.1)

$$
\rho(\vec{r})=\frac{1}{2}(I+\vec{r} \cdot \vec{\sigma})
$$

where $\vec{r}=(r \sin \theta \cos \phi, r \sin \theta \sin \phi, r \cos \theta)$ and $0 \leq r \leq 1,0 \leq \theta \leq \pi, 0 \leq \phi \leq 2 \pi$. The state is a pure one $|\psi\rangle=\cos \frac{\theta}{2}|0\rangle+e^{i \phi} \sin \frac{\theta}{2}|1\rangle$ for $r=1$ (on the sphere), or a mixed one for $r<1$ (inside the sphere). Specially, it is a maximally-mixed one $\frac{1}{2}(|0\rangle\langle 0|+| 1\rangle\langle 1|)$ for $r=0$ (zero vector).

In RSP protocol for pure states, a state subset $\chi$ is pre-agreed by Alice and Bob. Particles $A$ and $B$, shared by Alice and Bob, are in maximally entangled state

$$
\left|\Psi^{-}\right\rangle_{A B}=\frac{1}{\sqrt{2}}\left(|0\rangle_{A}|1\rangle_{B}-|1\rangle_{A}|0\rangle_{B}\right),
$$


and Alice wants to help Bob prepare a state $|\psi\rangle \in \chi$ in the distance. Here $|\psi\rangle$ is selected from $\chi$ randomly by Alice and is unknown to Bob. For example, $\chi$ can be the equatorial or polar great circle on the Bloch sphere and $|\psi\rangle$ is randomly selected from it.

To complete RSP, we can expand $\left|\Psi^{-}\right\rangle_{A B}$ in the basis $\left\{|\psi\rangle,\left|\psi_{\perp}\right\rangle\right\}$,

$$
\left|\Psi^{-}\right\rangle_{A B}=\frac{1}{\sqrt{2}}\left(|\psi\rangle_{A}\left|\psi_{\perp}\right\rangle_{B}-\left|\psi_{\perp}\right\rangle_{A}|\psi\rangle_{B}\right)
$$

where $\left|\psi_{\perp}\right\rangle=e^{i \phi} \sin \frac{\theta}{2}|0\rangle-\cos \frac{\theta}{2}|1\rangle$. Alice would perform a von Neumann measurement $\left\{|\psi\rangle,\left|\psi_{\perp}\right\rangle\right\}$ on her particle $A$ and send the result $0 / 1\left(|\psi\rangle /\left|\psi_{\perp}\right\rangle\right)$ to Bob. Or she can $(i)$ perform a unitary rotation $U(\theta, \phi)^{\dagger}$ on $A$,

$$
U_{A}(\theta, \phi)^{\dagger}\left|\Psi^{-}\right\rangle_{A B}=\frac{1}{\sqrt{2}}\left(|0\rangle_{A}\left|\psi_{\perp}\right\rangle_{B}-|1\rangle_{B}|\psi\rangle_{B}\right)
$$

where

$$
U(\theta, \phi)=\left(\begin{array}{cc}
\cos \theta / 2 & -e^{-i \phi} \sin \theta / 2 \\
e^{i \phi} \sin \theta / 2 & \cos \theta / 2
\end{array}\right)
$$

(ii) carry out a von Neumann measurement $\{|0\rangle,|1\rangle\}$; and (iii) send the result $0 / 1(|0\rangle /|1\rangle)$ to Bob (see Fig.2a). If the result is 1 , Bob will find his particle $B$ in $|\psi\rangle$, which is the state Alice wants to prepare. For the result 0 , $B$ will be in $\left|\psi_{\perp}\right\rangle$ and an operation is need for Bob to flip $\left|\psi_{\perp}\right\rangle$ into $|\psi\rangle$. Generally, such operation is unavailable because the universal NOT gate is forbidden for Bob has no knowledge of the state. However, it is possible for some special $\chi$, such as $\sigma_{Z}$ for the polar greatest circle and $i \sigma_{Y}$ for the equatorial circle. In the rest of this paper, we only discuss the situation that Alice obtain the result 1.

In [7], Berry and Sanders have generalized the protocol to mixed state preparation by using POVM instead of von Neumann measurement. The mixed state to be prepared (see eq.(1)) can be decomposed into

$$
\rho(\vec{r})=\frac{1+r}{2}|\psi\rangle\left\langle\psi\left|+\frac{1-r}{2}\right| \psi_{\perp}\right\rangle\left\langle\psi_{\perp}\right|
$$

To complete RSP of $\rho(\vec{r})$, Alice would carry out a two-element POVM $\left\{\Pi^{0}, \Pi^{1}\right\}$ instead of $\{|0\rangle,|1\rangle\}$,

$$
\Pi^{1}=\frac{1-r}{2}|0\rangle\left\langle 0\left|+\frac{1+r}{2}\right| 1\right\rangle\langle 1|, \Pi^{0}=I-\Pi^{1} .
$$

If Alice gets the result $1, B$ will be in $\rho(\vec{r})$. The essence is the realization of POVM. Here, the POVM can be performed by an auxiliary qubit and Controlled - NOT operation. The whole process of RSP can be divided into the following five steps (see Fig.2b). 1) a unitary rotation $U(\theta, \phi)^{\dagger}$ on $A ; 2$ ) a Controlled - NOT operation $U_{C N O T}$ where $A$ is the controller and the auxiliary qubit $a$ in initial state $|0\rangle$ is the target; 3) another unitary rotation $U_{A}(r)$ on $A$; 4 ) a von Neumann measurement on $A$; and 5$)$ sending the result to Bob. And it can be represented as

$$
\begin{aligned}
& \left|\Psi^{-}\right\rangle_{A B}|0\rangle_{a} \stackrel{U_{A}(\theta, \phi)^{\dagger}}{\rightarrow} \frac{1}{\sqrt{2}}\left(|0\rangle_{A}\left|\psi_{\perp}\right\rangle_{B}-|1\rangle_{B}|\psi\rangle_{B}\right)|0\rangle_{a} \\
& \stackrel{U_{C N O T}(A \rightarrow a)}{\rightarrow} \frac{1}{\sqrt{2}}\left(|0\rangle_{A}\left|\psi_{\perp}\right\rangle_{B}|0\rangle_{a}-|1\rangle_{B}|\psi\rangle_{B}|1\rangle_{a}\right) \\
\stackrel{U_{A}(r)}{\rightarrow}|\varphi\rangle_{A B a}= & \frac{1}{2}\left(\sqrt{1+r}|0\rangle_{A}\left|\psi_{\perp}\right\rangle_{B}|0\rangle_{a}+\sqrt{1-r}|0\rangle_{A}|\psi\rangle_{B}|1\rangle_{B}\right. \\
& \left.+\sqrt{1-r}|1\rangle_{A}\left|\psi_{\perp}\right\rangle_{B}|0\rangle_{a}-\sqrt{1+r}|1\rangle_{A}|\psi\rangle_{B}|1\rangle_{a}\right),
\end{aligned}
$$

where

$$
U(r)=\left(\begin{array}{cc}
\sqrt{\frac{1+r}{2}} & -\sqrt{\frac{1-r}{2}} \\
\sqrt{\frac{1-r}{2}} & \sqrt{\frac{1+r}{2}}
\end{array}\right) .
$$

For the result $1, B$ will be in

$$
\rho_{B}=\frac{\operatorname{Tr}_{A a}\left[|1\rangle_{A A}\langle 1|| \varphi\rangle_{A B a A B a}\langle\varphi|\right]}{\operatorname{Tr}_{A B a}\left[|1\rangle_{A A}\langle 1|| \varphi\rangle_{A B a A B a}\langle\varphi|\right]} .
$$

It's easy to see that the protocol is valid for pure state by setting $r=1$. 


\section{EFFECTS OF NOISY ENTANGLEMENT}

All the discussions above, both pure and mixed states, are based on the maximally entangled states shared between Alice and Bob. Due to the interaction with the environment, the entanglement will be partially destroyed. Such decoherence is possible during the distribution or storage of entanglement, So the final result of RSP will be influenced.

Generally, all physical processes, including decoherence evolution, can be represented by a complete positive map. Suppose Alice and Bob share an entangled state after decoherence,

$$
\rho_{A B}=\widehat{S}\left(\left|\Psi^{-}\right\rangle_{A B A B}\left\langle\Psi^{-}\right|\right)
$$

where $\widehat{S}$ is the operator of decoherence evolution. The final state that Bob obtains after the five steps above $(1-5)$ will be

$$
\rho_{B}=\frac{\operatorname{Tr}_{A a}\left[|1\rangle_{A A}\langle 1| \varrho_{A B a}\right]}{\operatorname{Tr}_{A B a}\left[|1\rangle_{A A}\langle 1| \varrho_{A B a}\right]}
$$

where

$$
\varrho_{A B a}=U_{A}(r) \otimes U_{C N O T}(A: a) \otimes U_{A}(\theta, \phi)^{\dagger} \rho_{A B} U_{A}(\theta, \phi) \otimes U_{C N O T}(A: a)^{\dagger} \otimes U_{A}(r)^{\dagger} .
$$

The effects of decoherence on RSP can be denoted by the state fidelity between $\rho_{B}$ and $\rho(\vec{r})$ (to be prepared),

$$
F\left(\rho(\vec{r}), \rho_{B}\right)=\operatorname{Tr}\left[\sqrt{\rho(\vec{r})^{1 / 2} \rho_{B} \rho(\vec{r})^{1 / 2}}\right]
$$

Here, two types of decoherence are considered, depolarizing and dephasing.

The entangled state after depolarizing is

$$
\rho_{A B}^{\prime}(p)=p\left|\Psi^{-}\right\rangle_{A B A B}\left\langle\Psi^{-}\right|+(1-p) \frac{I_{A}}{2} \otimes \frac{I_{B}}{2},
$$

which can be considered as a mixture of maximal entanglement and maximal mixed state. The result state that Bob gets is

$$
\rho_{B}^{\prime}=\frac{1+p r}{2}|\psi\rangle\left\langle\psi\left|+\frac{1-p r}{2}\right| \psi_{\perp}\right\rangle\left\langle\psi_{\perp}\right|
$$

and the state fidelity is

$$
F\left(\rho(\vec{r}), \rho_{B}^{\prime}\right)=\frac{1}{2}(\sqrt{(1+r)(1+p r)}+\sqrt{(1-r)(1-p r)})
$$

Specially, for maximal entanglement shared $p=1, F=1$; for pure states to be prepared $r=1, F=\sqrt{\frac{1+p}{2}}$; and for maximally-mixed states $r=0, F=1$. We found that the fidelity is independent of $\theta$ and $\phi$ and only related to the quantum channel $(p)$ and $r$.

For dephasing decoherence,

$$
\rho_{A B}^{\prime \prime}(p)=p\left|\Psi^{-}\right\rangle_{A B A B}\left\langle\Psi^{-}\right|+\frac{1-p}{2}\left(|0\rangle_{A A}\langle 0|\otimes| 1\rangle_{B B}\langle 1|+| 1\rangle_{A A}\langle 1|\otimes| 0\rangle_{B B}\langle 0|\right) .
$$

It can be considered as a mixture of maximal entanglement and classical correlation. The result state and the fidelity of RSP are

$$
\rho_{B}^{\prime \prime}=\frac{1+p}{2}\left(\frac{1+r}{2}|\psi\rangle\left\langle\psi\left|+\frac{1-r}{2}\right| \psi_{\perp}\right\rangle\left\langle\psi_{\perp}\right|\right)+\frac{1-p}{2}\left(\frac{1+r}{2}\left|\psi^{\prime}\right\rangle\left\langle\psi^{\prime}\left|+\frac{1-r}{2}\right| \psi_{\perp}^{\prime}\right\rangle\left\langle\psi_{\perp}^{\prime}\right|\right)
$$

and

$$
F\left(\rho(\vec{r}), \rho_{B}^{\prime \prime}\right)=\sqrt{\left(\frac{\alpha+\beta}{2}\right)+\sqrt{\left(\frac{\alpha-\beta}{2}\right)^{2}+\gamma^{2}}}+\sqrt{\left(\frac{\alpha+\beta}{2}\right)-\sqrt{\left(\frac{\alpha-\beta}{2}\right)^{2}+\gamma^{2}}}
$$


where

$$
\begin{aligned}
\left|\psi^{\prime}\right\rangle & =\cos \frac{\theta}{2}|0\rangle-e^{i \phi} \sin \frac{\theta}{2}|1\rangle,\left|\psi_{\perp}^{\prime}\right\rangle=e^{i \phi} \sin \frac{\theta}{2}|0\rangle+\cos \frac{\theta}{2}|1\rangle, \\
\alpha & =\frac{1}{8}\left((1+p)(1+r)^{2}+(1-p)(1+r)(1+r \cos 2 \theta)\right) \\
\beta & =\frac{1}{8}\left((1+p)(1-r)^{2}+(1-p)(1-r)(1-r \cos 2 \theta)\right) \\
\gamma & =\frac{1}{8} r(1-p) \sqrt{1-r^{2}} \sin 2 \theta .
\end{aligned}
$$

Specially, for maximal entanglement shared $p=1, F=1$; for pure states $r=1, F=\frac{1}{2} \sqrt{3+p+(1-p) \cos 2 \theta}$; and for $r=0, F=1$.

Here the fidelity for depolarizing is found to be independent on states to be prepared. While for dephasing, it depends only on the proportion of $|0\rangle$ and $|1\rangle$, i.e. depending on $\theta$, but not on the relative phase $\phi$. And, obviously, the later is better than former, which means classical correlation can help remote state preparation.

\section{EXPERIMENTS OF REMOTE STATE PREPARATION FOR SINGLE PHOTONS}

In this section, an experiment of remote state preparation for single photons is carried out by using spontaneous parametric down conversion (SPDC) and linear optical elements. Here, we only discuss the RSP in dephasing noisy channel. The setup is represented in Fig.3. A pulse of ultraviolet (UV) light pass through a BBO crystal $(0.5 \mathrm{~mm}$, cut for type-II phase match). The UV pulse is frequency-doubled pulse (less than $200 \mathrm{fs}$ with $82 \mathrm{MHz}$ repetition and $390 \mathrm{~nm}$ center-wavelength) from a mode-locked Ti: sapphire laser (Tsunami by Spectra-Physics) . Because of the birefringence of ordinary light ( $o$ light) and extra-ordinary light ( $e$ light) in BBO crystal, the state of the biphoton from SPDC process is no longer the maximal entangled one, but is the the state like

$$
\rho_{A B}(p)=p\left|\Psi^{-}\right\rangle_{A B A B}\left\langle\Psi^{-}\right|+\frac{1-p}{2}\left(|H\rangle_{A A}\langle H|\otimes| V\rangle_{B B}\langle V|+| V\rangle_{A A}\langle V|\otimes| H\rangle_{B}{ }_{B}\langle H|\right) .
$$

Where $p$ can be adjusted by quartz plate with different thickness. In our experiment, we only chose $p=0.9$ and 0.7 . When $p=0.9$, we use the technology of tomography to reconstruct density matrix of the state from SPDC process [14],

$$
\left(\begin{array}{llll}
0.001875 & -0.018531+0.013719 i & 0.002594+0.017125 i & 0.01-0.015437 i \\
-0.018531+0.013719 i & 0.50125 & -0.435688+0.002406 i & -0.007469+0.007281 i \\
0.002594+0.017125 i & -0.435688+0.002406 i & 0.494375 & -0.007281+0.005813 i \\
0.01+0.015438 i & -0.007469+0.007281 i & -0.007281+0.005813 i & 0.0025
\end{array}\right) .
$$

The fidelity between $\rho_{A B}(0.9)$ and the upper state we get experimently is $99.7 \%$. And also, we get the state from SPDC process when $p=0.7$, the fidelity is $99.5 \%$. We have possessed the perfect quantum channel (noisy entanglement), and next, we can carry out our schemes of remote state preparation.

In Fig.3, photon A passes through path 2. First, it is rotated by QWP1 and HWP1 which realize the unitary operator $U_{A}(\theta, \phi)^{\dagger}$ (see eq. (5)). Second, photon A transmits a $3.0 \mathrm{~mm} \beta$-BBO crystal, Whose $o$ axis is fixed horizontally and $e$ axis is fixed vertically. Here we chose the time degrees of freedom of photon A passing through BBO crystal to be the auxiliary qubit $\left(\left|t_{o}\right\rangle\right.$ for ordinary light and $\left|t_{e}\right\rangle$ for extra-ordinary light). After $3.0 \mathrm{~mm}$ BBO crystal, the separation of wavepackets between $\mathrm{H}(o)$ - and $\mathrm{V}(e)$-polarized light is about $350 \mu \mathrm{m}$. Because the coherent length of the wavepacket is about $150 \mu \mathrm{m}$ (4 $\mathrm{nm}$ FWHM interference filter is inserted before each detector), There is is no superposed parts between the $\mathrm{H}(o)$ - and $\mathrm{V}(e)$-polarized light. So a controlled-NOT operation is accomplished after the photon A passes through the $\mathrm{BBO}$ crystal

$$
(a|H\rangle+b|V\rangle)\left|t_{0}\right\rangle \rightarrow a|H\rangle\left|t_{o}\right\rangle+b|V\rangle\left|t_{e}\right\rangle
$$

where the polarization, $|H\rangle(|0\rangle) /|V\rangle(|1\rangle)$, is the control qubit and the time, $\left|t_{o}\right\rangle(|0\rangle) /\left|t_{e}\right\rangle(|1\rangle)$, is the target qubit. In our experiment, the input target qubit is fixed in $\left|t_{o}\right\rangle(|0\rangle)$. Following $3.0 \mathrm{~mm} \mathrm{BBO}$, photon A is operated by HWP2 which realizes the unitary $U_{A}(r)$ (see eq.(9)). The time qubit is traced when the photon is detected by a single photon detector. Photon B passes through path 1. Any single-qubit mixed states Alice want to prepare can be remotely prepared in Bob's laboratory by adjusting the angles of QWP1, HWP1 and HWP2 15] at Alice's side, and Bob can use technology of quantum state tomography to reconstruct the density matrix of it. 
To prepare pure states, we fixed the angle of HWP2 $0(r=1)$. QWP1 and HWP1 can help Alice remotely prepare any pure states in Bob's laboratory. Bob use technology of tomography to reconstruct the state matrix of it again.

In our experiment, several pre-agreed state sets are selected (see in Fig.1). For each pre-agreed state set, we finish the process of RSP when $p=0.9$ and 0.7 respectively as shown in Fig.4 and Fig.5. Three sets of pure states are remotely prepared as show in Fig 4.: 1)the polar great circle on the sphere crossed by X-Z plane $(r=1, \theta \in[0, \pi]$, $\phi=0), 2)$ the polar great circle by Y-Z plane $(r=1, \theta \in[0, \pi], \phi=\pi / 2)$, and 3)the equatorial great circle by X-Y plane $(r=1, \theta=\pi / 2, \phi \in[0,2 \pi])$. It is found that the fidelity of RSP is independent of the relative phase $\phi$ and depend on $\theta$ and the noise of entanglement, as eq.(20) shows. Four sets of mixed states are shown in Fig.5a and 5b: 4)a small circle on the X-Z plane $\left(r=\cos ^{2} \frac{\pi}{8}, \theta \in[0, \pi], \phi=0\right)$; 5)the zero vector $\vec{O}$; and two lines on the X-Z plane: 6) $(r \in[-1,1], \theta=\pi / 4, \phi=0)[16]$ and 7$)(r \in[-1,1], \theta=\pi / 2, \phi=0)[16]$. Fig.5 tell us that the the fidelity of RSP of mixed state is the cosine function of $\theta$, and the fidelity of the maximally mixed state is always one(Fig.5a). the effect of noise to pure states is greater than mixed states (Fig.5b). In Fig.4 and Fig.5, the solid lines are the theoretical results given by eq.(20). For example, in Fig.4b, the state from the polar great circle on the sphere crossed by X-Z plane $(r=1, \theta \in[0, \pi], \phi=0)$ are remotely prepared and the parameter of channel $p=0.7$, So the fidelity curve given by eq.(20) is $F=\frac{1}{2} \sqrt{3.7+0.3 \cos 2 \theta}$. It is found that all experiment results (the square dots) match the theoretical prediction (the solid lines) well. The imperfection of our experimental results only comes from the fluctuation of the coincidence counts and the limited precision $\left(2^{\circ}\right)$ of waveplates (HWPs and QWPs) 14].

\section{CONCLUSION}

In conclusion, we present a practical and general scheme of remote preparation for pure and mixed states. An auxiliary qubit and controlled-NOT operation are used in the scheme. The effects of noisy entanglement are discussed for two important types of decoherence, depolarizing and dephasing, by the state fidelity. The fidelity for depolarizing is found to be independent of $\theta$ and $\phi$ of states to be prepared and only related to the quantum channel $(p)$ and $r$. While for dephasing, it depends only on the proportion of $|0\rangle$ and $|1\rangle$, i.e. depending on $\theta$, but not on the relative phase $\phi$. And the dephasing entanglement is always better than the depolarizing one for RSP, which implies classical correlation is helpful for RSP. In our experiment, we successfully complete RSP of pure and mixed states via dephasing entanglement by using spontaneous parametric down conversion (SPDC) and linear optical elements. Our scheme can be carried out remotely by using optic fiber or in free space.

\section{Acknowledgment}

We wish to thank Yong-Sheng Zhang, Guo-Ping Guo and Ming-Yong Ye for interesting and helpful discussion. This work was funded by the National Fundamental Research Program (2001CB309300), the Innovation Funds from Chinese Academy of Sciences, and also by the outstanding Ph. D thesis award and the CAS's talented scientist award rewarded to Lu-Ming Duan.

[1] M. A. Nielsen and I. L. Chuang, Quantum Computation and Quantum Information (Cambridge University Press, Cambridge, England, 2000)

[2] C. H. Bennett, et al. Phys. Rev. Lett. 70, 1895 (1993). D. Bouwmeester, J. W. Pan, K. Mattle, M. Eibl, H. Weinfurter, and A. Zeilinger, Nature (London) 390, 575 (1997).

[3] H. K. Lo, Phys. Rev. A 62, 012313 (2000)

[4] A. K. Parti, Phys. Rev. A 63, 014302 (2001)

[5] C. H. Bennett, D. P. DiVincenzo, P. W. Shor, J. A. Smolin, B. M. Terhal, and W. K. Wootters, Phys. .Rev. Lett, 87, 077902 (2001).

[6] I. Devetak and T. Berger, Phys. Rev. Lett, 87, 197901 (2001).

[7] D. W. Berry and B. C. Sanders, Phys. Rev. Lett, 90, 057901 (2003).

[8] D. W. Leung and P. W. Shor, Phys. Rev. Lett, 90, 127905 (2003).

[9] X. H. Peng, X. W. Zhu, X. M. Fang, M. Feng, M. L. Liu, and K. L. Gao, Phys. Lett. A 306, 271-276 (2003).

[10] A. K. Pati, quant-ph/0212164

[11] M. Y. Ye, Y. S. Zhang, and G. C. Guo, Phys. Rev. A 69, 022310 (2004)

[12] C. H. Bennett, P. Hayder, D. W. Leung, P. W. Shor, and A. Winter, quant-ph/0307100

[13] A. Abeyesingle, and P. Hayden, Phys. Rev. A 69, 062319 (2003)

[14] D. F. V. James, P. G. Kwait, W. J. Munro, and A. G. White, Phys. Rev. A, 64, 052312 (2001)

[15] P. G. Kwiat, J. B. Altepeter, D. A. Branning, E. R. Jeffrey, N. A. Peters, T.C. Wei, quant-ph/0303040 
[16] Here we write $(r \in[0,1], \theta=\pi / 2, \phi=0) \bigcup(r \in[0,1], \theta=3 \pi / 2, \phi=0)$ as $(r \in[-1,1], \theta=\pi / 2, \phi=0)$ and write $(r \in[0,1], \theta=\pi / 4, \phi=0) \bigcup(r \in[0,1], \theta=5 \pi / 4, \phi=0)$ as $(r \in[-1,1], \theta=\pi / 4, \phi=0)$ for the convenience.

Figure Caption

Figure 1. The Bloch sphere. The points on and in it represent the states of a single qubit and vector $\vec{r}$ represent the position of the points.

Figure 2. Schematic protocols for remote preparation of a) pure state b) mixed state. The EPR state pre-shared by Alice and Bob.

Figure 3. Experimental setup for remote state preparation in dephasing channel. Alice operates and measures the photon in path 2, and Bob reconstruct the state of the photon in path 1 by using the technology of quantum state tomography. HWP and QWP represent the half wave plate and quarter wave plate respectively. PA is the polarization analyzer, and IF is the $4 \mathrm{~nm}$ FWHM interference filter is used before each detector.

Figure 4. Fidelity of RSP of pure states from different ensembles: 1) In a and b, the polar greatest circle on the sphere in X-Z plane; 2) In c and d, the polar greatest circle on the sphere in Y-Z plane; 3) In e and f, the states on the equatorial greatest circle.

Figure 5. Fidelity of RSP of mixed states from different ensembles: a), the states are from a small circle on the X-Z plane $\left(r=\cos ^{2} \frac{\pi}{8}, \theta \in[0,2 \pi], \phi=0\right)$ in the two figures above and are maximally mixed states; b), the states in the above and below two figures are from the two lines in X-Z plane: $(r \in[-1,1], \theta=\pi / 4, \phi=0)$ and $(r \in[-1,1], \theta=\pi / 2, \phi=0)$ respectively. 


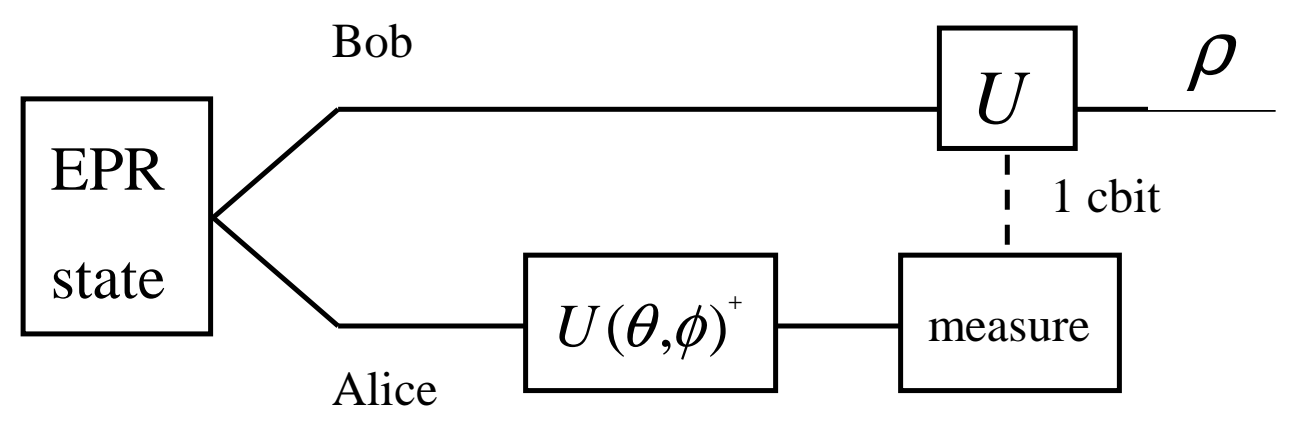




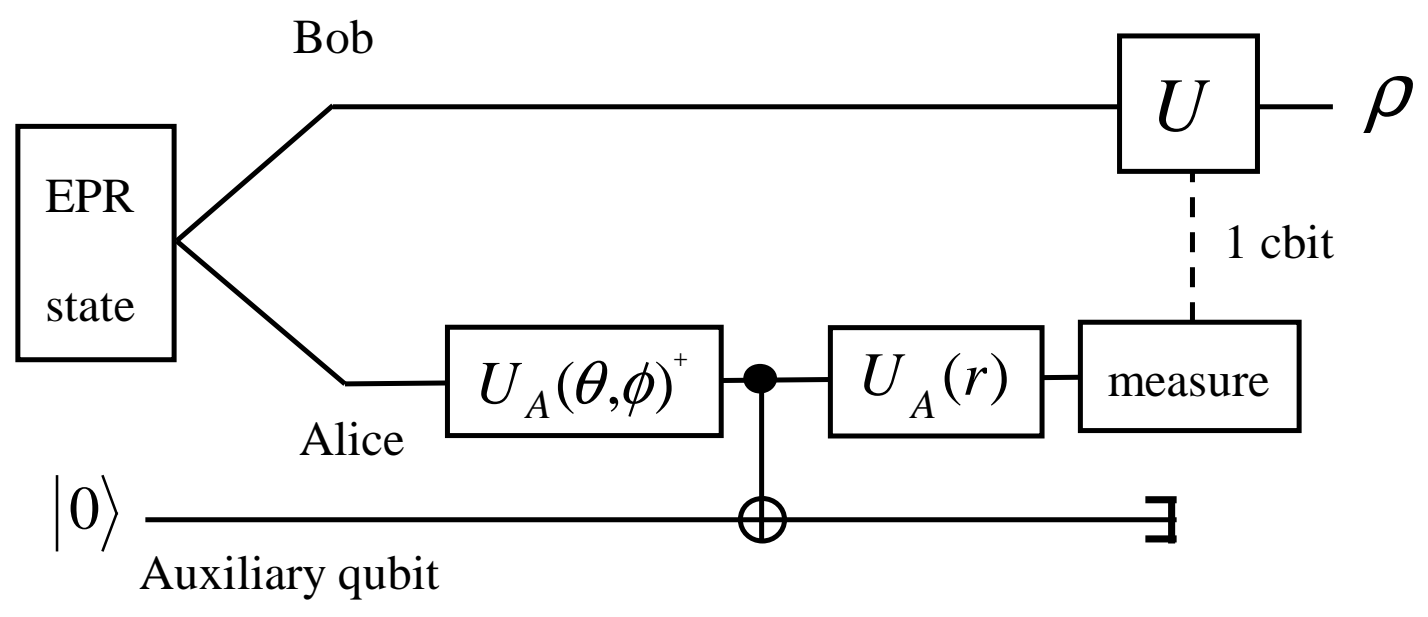




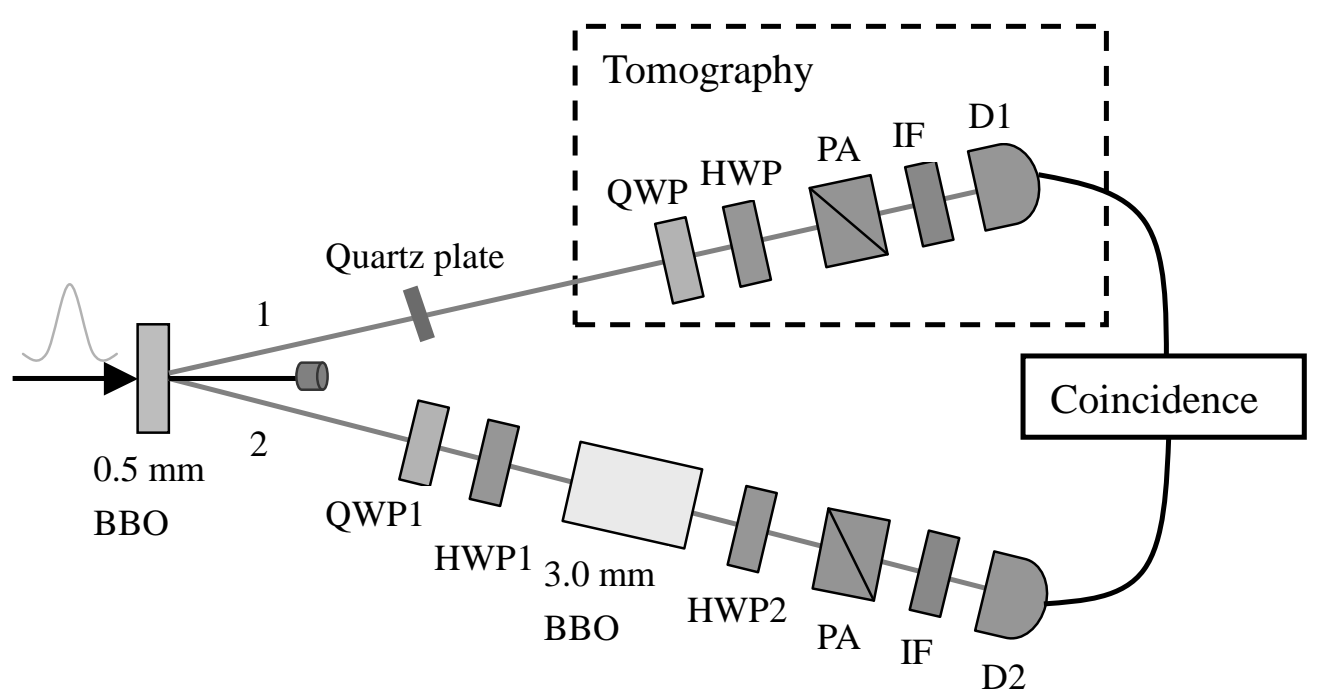




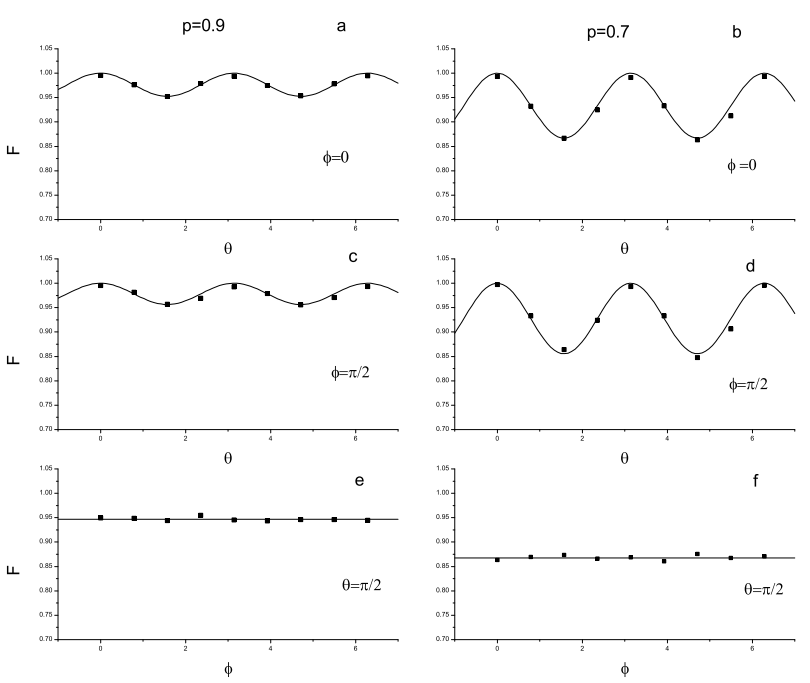




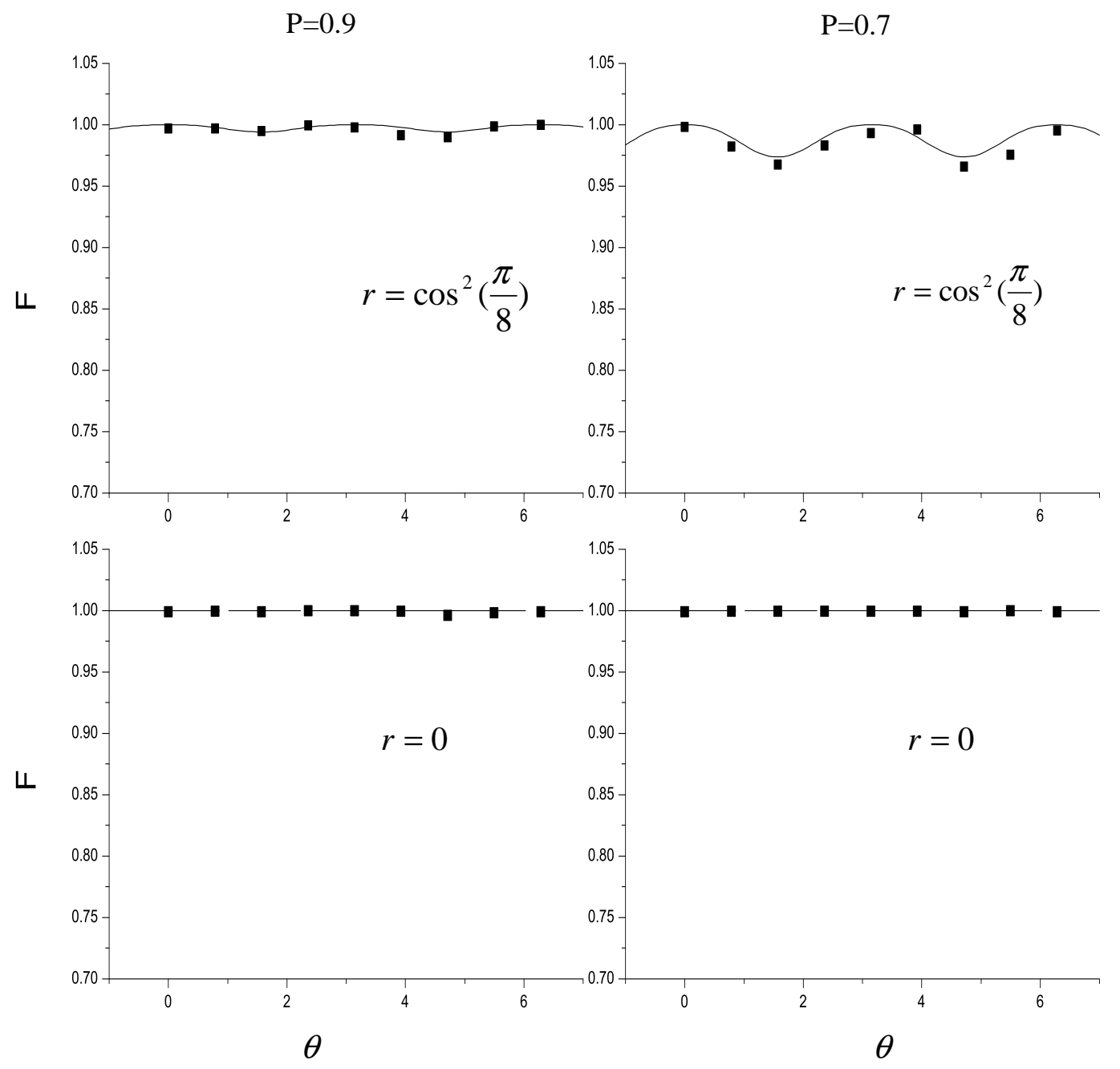




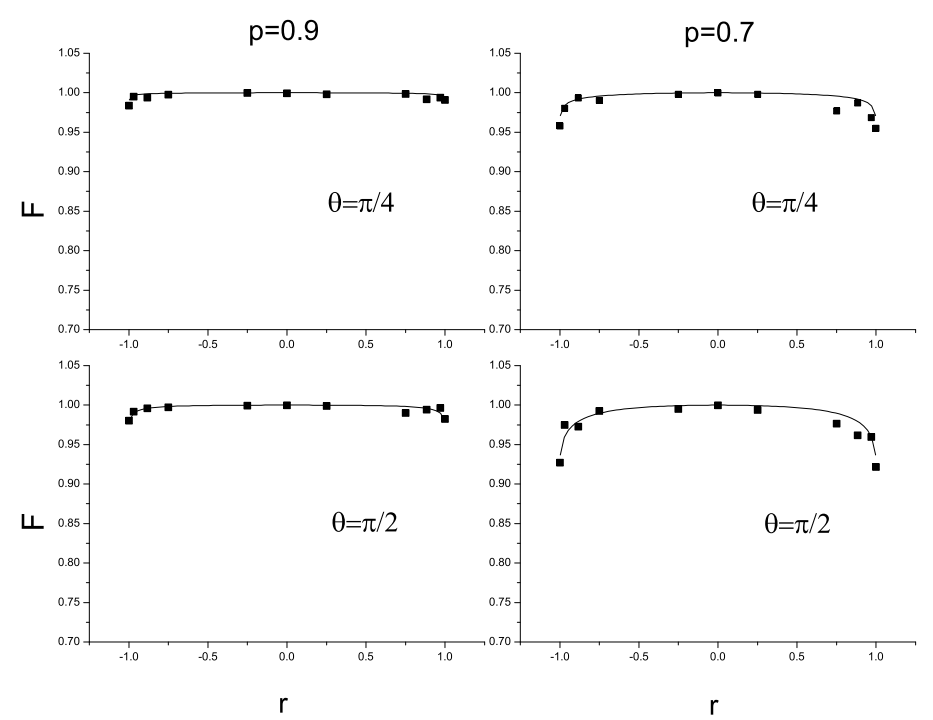

